\title{
Vasculitis leucocitoclástica cutánea en paciente con cáncer gástrico como síndrome paraneoplásico. Reporte de caso
}

\author{
Cutaneous leukocytoclastic vasculitis and gastric adenocarcinoma as paraneoplastic \\ syndrome. Case report
}

\footnotetext{
- Natallie Jurado1, Jesús Sánchez²

Médica internista. Hospital Universitario San Ignacio (Bogotá).

Médico especialista en oncología clínica Centro Javeriano de Oncología, Pontificia Universidad Javeriana.
}

\section{Resumen}

La vasculitis más frecuentemente relacionada con neoplasia es una vasculitis de pequeño vaso, la vasculitis leucocitoclástica. Se asocia en su mayoría a neoplasias hematolinfoides y no a tumores sólidos, y se considera que la inflamación cutánea es el resultado del depósito de complejos inmunes circulantes contra los antígenos tumorales depositados en la pared del vaso sanguíneo, que, junto con la activación del complemento, llevan a inflamación vascular. En un tercio de los casos, la vasculitis leucocitoclástica precede a la aparición de la neoplasia en semanas e incluso meses. Se reporta un caso de vasculitis leucocitoclástica cutánea en una paciente con cáncer gástrico metastásico en progresión.

Palabras clave: vasculitis leucocitoclástica cutánea, cáncer gástrico, síndrome paraneoplásico, ácido valproico.

\begin{abstract}
The most common vasculitis associated with malignancy is inflammation of small vessels that most commonly presents as cutaneous leukocytoclastic vasculitis. Hematologic malignancies are more commonly associated than solid tumors and the development of cutaneous inflammation is the result of circulating immune complexes containing tumor antigens deposited along the vascular walls, leading to complement activation and vascular inflammation. About one third of leukocytoclastic vasculitis cases can precede the clinical appearance of cancer by weeks to months. We report a case of a patient with metastasic gastric cancer with progression and cutaneous leukocytoclastic vasculitis associated.
\end{abstract}

Key words: Cutaneous leukocytoclastic vasculitis, gastric cancer, paraneoplastic syndrome, valproic acid.

\section{Introducción}

La vasculitis cutánea es un grupo de síndromes heterogéneos caracterizados por el compromiso de la piel, con hallazgos histopatológicos de inflamación y daño de vasos sanguíneos. Por sí sola constituye un proceso benigno, consecuencia de infecciones, hipersensibilidad, enfermedades autoinmunes, medicamentos, o puede corresponder a una dermatosis asociada a malignidad y comportarse como un síndrome paraneoplásico que puede preceder, coincidir o aparecer después del diagnóstico de cáncer; la vasculitis leucocitoclástica cutánea (VLC) es la vasculitis paraneoplásica más frecuente, se relaciona con neoplasias hematológicas en el 70-90\% de los casos y el pronóstico depende de la neoplasia de base1.

Se reporta un caso de VCL en una paciente con cáncer gástrico metastásico en progresión con resolución de lesiones en piel asociado al control de la enfermedad neoplásica con segunda línea de quimioterapia. Se realiza revisión de la literatura sobre vasculitis leucitoclástica como síndrome paraneoplásico.

Método: revisión de base de datos PubMed.

Recibido: 10 de abril de 2017; aceptado: 15 de septiembre de 2017 


\section{Descripción del caso}

Paciente femenina de 53 años procedente de Bogotá con antecedente de epilepsia en la infancia secundaria a meningitis controlada con ácido valproico $750 \mathrm{mg} /$ día y antecedente de tabaquismo activo 9 paquetes/año. Ingresa a la institución por dolor epigástrico de tres meses de evolución 5/10, emesis posprandial, pérdida de peso, astenia, adinamia, con endoscopia de vías digestivas altas que muestra lesión ulcerada de aspecto neoplásico, la cual compromete cuerpo, antro y píloro; TAC (tomografía axial computarizada) abdominal que documenta extensión transmural, adenopatías en curvaturas mayor y menor, carcinomatosis peritoneal; y TAC de tórax normal. Se clasifica con Eastern Cooperative Oncology Group (ECOG) 1 e índice de Karnofsky del 80\%, y es llevada a gastroyeyunostomía paliativa por laparoscopia, con patología compatible con carcinoma mucinoso gástrico con componente de célula en anillo de sello estadio IV, Her-2 negativo. Se inicia quimioterapia paliativa esquema Xelox (capecitabina/oxaliplatino) seis ciclos, presentando náuseas, hiporexia, distensión abdominal, piel seca y fatiga posterior a quimioterapia, por lo cual se cambia esquema a capecitabina monoterapia dos ciclos, con evidencia de distensión abdominal y ascitis, se reinicia Xelox.

Presenta un mes después lesiones múltiples en piernas, muslos, abdomen y miembros superiores, con extensión progresiva, tipo máculas y pápulas parduzco-violáceas recurrentes, no dolorosas, que no desaparecen con digitopresión, sin compromiso de mucosas (figura 1), no asociadas a taquicardia ni

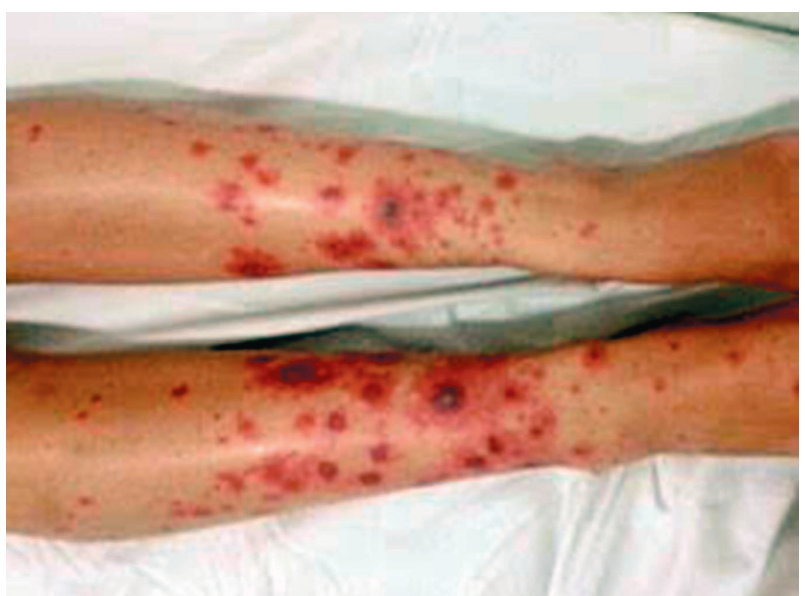

Figura 1. Lesiones múltiples en extremidades, abdomen, tipo máculas y pápulas parduzco-violáceas, no dolorosas, que no desaparecen con digitopresión, sin compromiso de mucosas (autorización previa de la paciente).
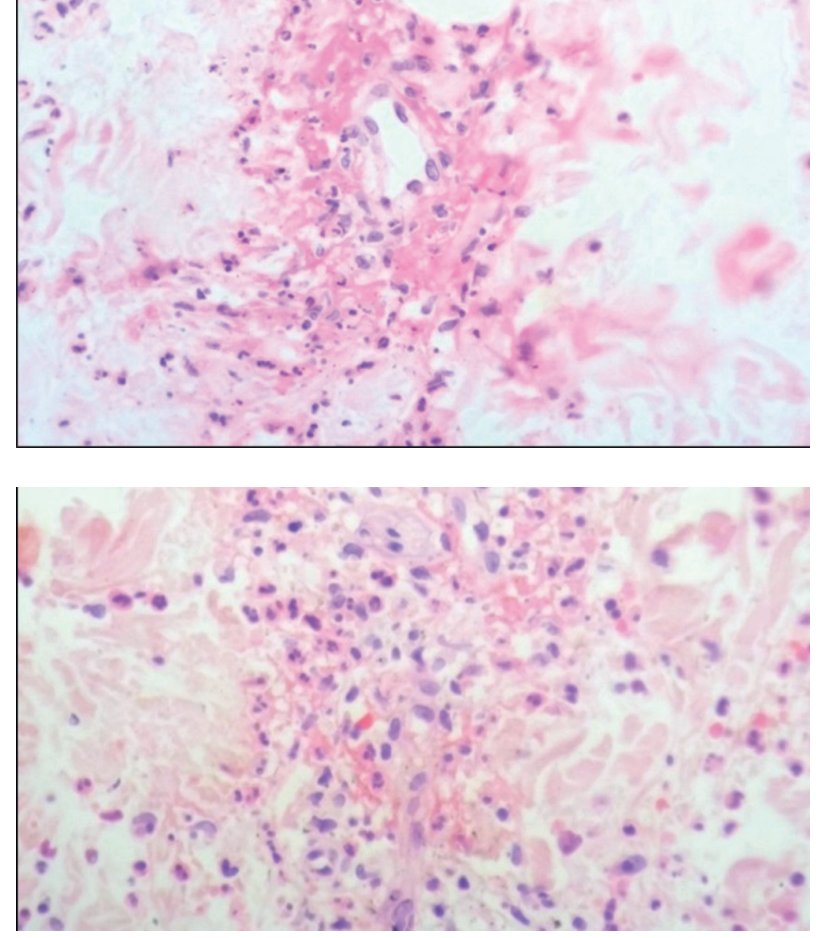

Figura 2. A. Infiltrado inflamatorio de predominio pericapilar neutrofílico en el endotelio vascular, con degeneración neutrofílica (leucocitoclasis). B. Infiltrado inflamatorio perivascular, con extravasación de eritrocitos.

taquipnea, no fiebre ni hipotermia, no síntomas en tracto respiratorio, urinario o foco infeccioso aparente, no leucocitosis ni neutrofilia, sin alteraciones en plaquetas, tiempos de coagulación, sin anemización, ni respuesta inflamatoria (leucocitos $6.470 \mathrm{x}$ $10^{3} / \mathrm{ul}$, neutrófilos $60,8 \%$, linfocitos $30,6 \%$, monocitos $7,98 \%$, eosinófilos $0,42 \%, \mathrm{Hb} 14 \mathrm{~g} / \mathrm{dl}$, plaquetas $193.800 \times 10^{3} / \mathrm{ul}$, VSG $40 \mathrm{~mm} / \mathrm{h}$ ), con transaminasas, bilirrubinas, lactato deshidrogenasa y fosfatasa alcalina normales, sin estigmas de autoinmunidad, por lo cual se descarta proceso infeccioso asociado o autoinmune y se sospecha probable vasculitis secundaria a medicamento versus neoplasia. Fue valorada por dermatología realizando biopsia con punch de piel, que documenta a nivel de dermis infiltrado inflamatorio predominantemente neutrofílico perivascular e intersticial, infiltración neutrofílica y necrosis fibrinoide en pared de pequeños vasos de dermis superficial y media, endotelio con cambios reactivos y extravasación de eritrocitos y leucocitoclastia (figura 2A). En la inmunofluorescencia, trazas de IgM y C3 en la pared de los vasos, con fibrinógeno fuertemente positivo, sin depósitos inmunes en membrana basal, compatible con vasculitis leucocitoclástica (figura 2B). Con este reporte inicialmente se considera secundaria a platinos, por lo cual se suspende tratamiento. 
Asiste a control dos meses después refiriendo, a pesar de no recibir platinos, reaparición de lesiones, persistencia de distensión abdominal y ascitis, por lo cual se sospechó asociación a fenómeno paraneoplásico, se ordena ciclo de esteroides con prednisolona $40 \mathrm{mg} / \mathrm{día}$ por tres días, antihistamínico, y se da orden para quimioterapia monoagente con irinotecán. Se evalúa en control al mes siguiente, refiriendo persistencia de lesiones en piel recurrentes a pesar de uso de esteroides dos ciclos y de suspensión de platinos, por lo cual se sospecha etiología paraneoplásica. Por trámites administrativos, inicia ciclo de irinotecán un mes luego de este control, con adecuada tolerancia, sin nuevos episodios de ascitis, sin dolor abdominal y resolución completa de lesiones en piel, sin recurrencias, con imágenes tomográficas de control a los tres meses sin nuevas lesiones, con compromiso imaginológico peritoneal sin cambios, completa 14 ciclos de irinotecán, con TAC de tórax y abdomen un año después, con enfermedad neoplásica estable.

\section{Discusión}

La vasculitis leucocitoclástica cutánea es una enfermedad inflamatoria de pequeño vaso caracterizada por inflamación, depósito de fibrina y restos nucleares en las vénulas poscapilares de la dermis superficial; cursa en el $40 \%$ de los casos con lesiones purpúricas palpables en miembros inferiores y síntomas generales leves, como fatiga, artralgias y fiebre, siendo menos común el compromiso renal o sistémico. Las lesiones en miembros superiores, tronco, cabeza y cuello son infrecuentes y pueden asociarse a una enfermedad más severa o a vasculitis sistémica ${ }^{1-3}$.

La vasculitis cutánea por sí sola es un proceso benigno, en el $40 \%$ de los casos idiopático, el $22 \%$ puede ser consecuencia de cualquier tipo de infección vírica, bacteriana, parasitaria o fúngica, especialmente del tracto respiratorio superior por Streptococcus haemolyticus grupo A, Staphylococcus aureus, cursando con bacteriemia, debido a la formación de autoanticuerpos; menos frecuente se ha asociado a Mycoplasma, sífilis y Mycobacterium tuberculosis. Por infecciones virales por formación de inmunocomplejos, como en hepatitis C y VIH (virus de inmunodeficiencia humana), también se han descrito casos de vasculitis cutánea en hepatitis $B$, herpes simple y citomegalovirus, en este último caso por daño endotelial directo; por infecciones micóticas por Candida Spp. secundario a la activación directa del sistema del complemento y menos frecuentemente por parásitos como Plasmodium ${ }^{4-6}$.

En el $20 \%$ de los casos, la vasculitis cutánea cursa como reacción adversa o de hipersensibilidad a medicamentos (en especial antiinflamatorios no esteroideos, penicilinas y tiazidas, menos frecuente antineoplásicos); un $12 \%$ se asocia a enfermedades del tejido conectivo, principalmente a lupus y artritis reumatoide; o puede corresponder a una dermatosis relacionada con malignidad, siendo la VLC la vasculitis paraneoplásica más frecuente $\mathrm{e}^{7-11}$.

La VLC se asocia en el $70-90 \%$ de los casos a neoplasias hematológicas, como linfomas, leucemia de células peludas, y menos frecuente a tumores sólidos, como cáncer de pulmón de célula no pequeña, próstata, colon, riñón, mama, carcinoma epidermoide de cabeza y cuello, endometrio y raramente en cáncer gástrico; usualmente la vasculitis y la neoplasia tienen un curso paralelo y normalmente las lesiones en piel aparecen después del diagnóstico de la neoplasia $3,7,10,11$

Los signos y síntomas de pacientes con vasculitis paraneoplásica son similares a los pacientes que no tienen cáncer, pero una edad mayor a 50 años al momento del diagnóstico, la falta de correlación con infecciones previas, medicamentos o enfermedades autoinmunes, un curso crónico recurrente, la elevación de la velocidad de sedimentación globular $>40$ mm/h (VSG), la asociación a citopenias, en especial anemia o la presencia de células inmaduras en sangre periférica, y la falta de respuesta a esteroides son hallazgos sugestivos de neoplasia oculta, además de los síntomas asociados a la neoplasia como ta|8,10,12-14.

El desarrollo de la enfermedad es secundario a las citoquinas pro inflamatorias producidas por las células tumorales y a los neoantígenos tumorales, los cuales estimulan la formación de complejos inmunes que se depositan en las paredes de los vasos sanguíneos; las células tumorales circulantes invaden la pared vascular generando daño endotelial mediado por complemento y se producen embolismos de células tumorales. En la histología se observa un infiltrado polimorfonuclear en vasos de pequeño calibre a nivel de la dermis superficial, leucocitoclastia, necrosis fibrinoide, extravasación 
de eritrocitos, y en la inmunofluorescencia se observan depósitos de complejos inmunes tipo lgG, IgM y C3 en la pared vascular2-3,7-13,15-18.

El tratamiento y pronóstico de las lesiones en piel puede o no responder a esteroides, mas presenta adecuada respuesta con el tratamiento efectivo del cáncer. En algunos casos puede requerir manejo con glucocorticoides en combinación con inmunosupresores tipo metrotexate, dapsona o azatioprina ${ }^{10}$.

\section{Conclusiones}

La vasculitis leucocitoclástica es la inflamación y daño de vasos sanguíneos en su mayoría idiopática una vez se descartan infecciones, en especial del tracto respiratorio superior, y por reacciones de hipersensibilidad, principalmente a medicamentos. En estos casos, el curso clínico suele ser un episodio único de lesiones en piel, autolimitado o de corta duración; con menor frecuencia la vasculitis es asociada a enfermedades del tejido conectivo, y como diagnóstico diferencial en menos del $5 \%$ de los casos se encuentra relacionada con malignidad, teniendo un curso crónico con lesiones que no remiten o con pobre respuesta a tratamiento, y su evolución se vincula a la de la enfermedad neoplásica de base.

Aunque la vasculitis es menos usual en pacientes con tumores sólidos versus hematolinfoides, dado que la paciente cursó con lesiones vasculíticas de curso crónico recurrentes sin respuesta inicial a esteroides y a suspensión de medicamentos, y sin otra sintomatología sugestiva de infección o autoinmunidad, se asoció a progresión de su enfermedad de base, confirmando la hipótesis de vasculitis paraneoplásica con la resolución de las lesiones con la nueva línea de tratamiento.

En este caso en particular, al realizar revisión de la literatura como hallazgo incidental, se encontró que el ácido valproico es un medicamento que induce la demetilación de ADN y la acetilación de histonas al inhibir las deacetilasas de histona (HDAC) clase I y clase II, el cual puede inhibir la proliferación celular e inducir la diferenciación de células tumorales, entre ellas las del cáncer gástrico; sin embargo, no hay reportes en la literatura de su uso en humanos con irinotecán ${ }^{19-23}$, por lo cual se requieren estudios para evaluar su efecto con diferentes líneas de quimioterapia y su probable impacto clínico.

\section{Referencias}

1. Loricera J, Calvo-Río V, Ortiz-Sanjuán F, González-López MA, Fernández-Llaca H, Rueda-Gotor J, et al. The spectrum of paraneoplastic cutaneous vasculitis in a defined population: incidence and clinical features. Medicine (Baltimore). 2013:92(6):331-43.

2. Yadav BS, Sharma SC, Kapoor RK. Paraneoplastic leukocytoclastic vasculitis in chronic lymphoid leukemia. J Cancer Res Ther. 2006;2(4):206-8

3. Quack H, Erpenbeck L, Wolff HA, Sprenger T, Seitz CS, Schön MP, et al. Oxaliplatin-induced leukocytoclastic vasculitis under adjuvant chemotherapy for colorectal cancer: two cases of a rare adverse event. Case Rep Oncol. 2013;6(3):609-15.

4. Marques C, Sereijo M, Domingues JC, Sequeira J, Poiares-Baptista AP. Vasculite leucocitoclásica. Revisao de 51 casos. Acta Med Port. 1995;8(1):15-22.

5. Pulido-Pérez A, Avilés-Izquierdo J, Suárez-Fernández R. Vasculitis cutáneas. Actas Dermosifiliogr. 2012;103(3):179-91.

6. Loricera J, González-Vela C, Blanco R, Hernández JL, Armesto S, González-López MA, et al. Histopathologic differences between cutaneous vasculitis associated with severe bacterial infection and cutaneous vasculitis secondary to other causes: study of 52 patients. Clin Exp Rheumatol. 2016;34(3 Suppl 97):S93-7.

7. Carlson JA. The histological assessment of cutaneous vasculitis Histopathology. 2010;56(1):3-23

8. Mebazaa A, Chouk S, Azzouz H, El Euch D, Rouhou RC, Trojjet $S$, et al. Leukocytoclastic vasculitis and gastric adenocarcinoma. Acta Dermatovenerol Alp Pannonica Adriat. 2010;19(1):21-3.

9. Gogia A, Kakar A, Bhalla S, Byotra SP. Leucocytoclastic vasculitis as a presentation of adenocarcinoma rectum. J Postgrad Med. 2005; 51(3):215-7.

10. Solans-Laqué $R$, Bosch-Gil JA, Pérez-Bocanegra C, SelvaO'Callaghan A, Simeón-Aznar CP, Vilardell-Tarres M. Paraneoplastic vasculitis in patients with solid tumors: report of $15 \mathrm{ca}-$ ses. J Rheumatol. 2008;35(2):294-304.

11. Sánchez-Guerrero J, Gutiérrez-Ureña $S$, Vidaller $A$, Reyes $E$, Iglesias A, Alarcón-Segovia D. Vasculitis as a paraneoplastic syndrome. Report of 11 cases and review of the literature. J Rheumatol. 1990;17(11):1458-62.

12. Fain $O$, Hamidou M, Cacoub P, Godeau B, Wechsler B, Pariès J, et al. Vasculitides associated with malignancies: analysis of sixty patients. Arthritis Rheum. 2007;57(8):1473-80.

13. García-Porrúa C, González-Gay MA. Cutaneous vasculitis as a paraneoplastic syndrome in adults. Arthritis Rheum. 1998;41(6):1133-5

14. Agha A, Bateman H, Sterrett A, Valeriano-Marcet J. Myelodysplasia and malignancy-associated vasculitis. Curr Rheumatol Rep. 2012;14(6):526-31.

15. Carlson JA, Chen KR. Cutaneous vasculitis update: small vessel neutrophilic vasculitis syndromes. Am J Dermatopathol. 2006;28(6):486-506.

16. Kathula SK, Thomas DE, Anstadt MP, Khan AU. Paraneoplastic cutaneous leukocytoclastic vasculitis and iron deficiency anemia as the presenting features of squamous cell lung carcinoma. J Clin Oncol. 2011;29(4):83-5.

17. Hill SE, Phillips R, Francis N, Agnew K. Small-vessel vasculitis following treatment with combination 5-fluorouracil/folinic acid and oxaliplatin. Clin Exp Dermatol. 2009;34(1):103-5. 
18. Al-Shamsi HO, Kee BK, Tetzlaff MT, Wolff RA. Capecitabineinduced leukocytoclastic vasculitis under neoadjuvant chemotherapy for locally advanced colorectal cancer. J Gastrointest Oncol. 2015;6(3):E40-3.

19. Yagi $Y$, Fushida S, Harada S, Kinoshita J, Makino I, Oyama K, et al. Effects of valproic acid on the cell cycle and apoptosis through acetylation of histone and tubulin in a scirrhous gastric cancer cell line. J Exp Clin Cancer Res. 2010;29:149.

20. Fushida S, Kaji M, Oyama K, Hirono Y, Nezuka H, Takeda T, et al. Randomized phase II trial of paclitaxel plus valproic acid vs paclitaxel alone as second-line therapy for patients with advanced gastric cancer. Onco Targets Ther. 2015;8:939-41.
21. Feng $W H$, Kenney SC. Valproic acid enhances the efficacy of chemotherapy in EBV-positive tumors by increasing lytic viral gene expression. Cancer Res. 2006;66(17):8762-9.

22. Hrzenjak A, Moinfar F, Kremser ML, Strohmeier B, Staber PB, Zatloukal K, et al. Valproate inhibition of histone deacetylase 2 affects differentiation and decreases proliferation of endometrial stromal sarcoma cells. Mol Cancer Ther. 2006;5(9):220310.

23. Göttlicher $M$, Minucci $S$, Zhu P, Krämer $\mathrm{OH}$, Schimpf $A$, Giavara $S$, et al. Valproic acid defines a novel class of HDAC inhibitors inducing differentiation of transformed cells. EMBO J. 2001;20(24):6969-78. 